\title{
Adaptive multiresolution approach for solution of hyperbolic PDEs
}

\author{
M.A. Alves ${ }^{\mathrm{a}, *}$, P. Cruz ${ }^{\mathrm{b}}$, A. Mendes ${ }^{\mathrm{b}}$, F.D. Magalhães ${ }^{\mathrm{b}}$, \\ F.T. Pinho ${ }^{\text {c }}$, P.J. Oliveira ${ }^{\mathrm{d}}$ \\ a Departamento de Engenharia Química, Faculdade de Engenharia da Universidade do Porto, \\ Rua Dr. Roberto Frias, 4200-465 Porto, Portugal \\ ${ }^{\mathrm{b}}$ Faculty of Engineering, LEPAE-Chemical Engineering Department, University of Porto, \\ Rua Dr. Roberto Frias, 4200-465 Porto, Portugal \\ c Centro de Estudos de Fenómenos de Transporte, DEMEGI, Faculdade de Engenharia da Universidade do Porto, \\ Rua Dr. Roberto Frias, 4200-465 Porto, Portugal \\ d Departamento de Engenharia Electromecânica, Universidade da Beira Interior, \\ Rua Marquês d'Ávila e Bolama, 6201-001 Covilhã, Portugal
}

Received 14 February 2002; accepted 25 April 2002

\begin{abstract}
This paper establishes an innovative and efficient multiresolution adaptive approach combined with high-resolution methods, for the numerical solution of a single or a system of partial differential equations. The proposed methodology is unconditionally bounded (even for hyperbolic equations) and dynamically adapts the grid so that higher spatial resolution is automatically allocated to domain regions where strong gradients are observed, thus possessing the two desired properties of a numerical approach: stability and accuracy.

Numerical results for five test problems are presented which clearly show the robustness and cost effectiveness of the proposed method.
\end{abstract}

(C) 2002 Elsevier Science B.V. All rights reserved.

Keywords: Hyperbolic equations; SMART high-resolution scheme; Multiresolution; Adaptive grid; Wavelet

\section{Introduction}

The numerical solution of partial differential equations (PDEs) that present steep moving fronts, in one or multiple dimensions, is an important subject of research in different areas. In this work we consider a class of non-linear advection-diffusion-reaction equations of the generic form

$$
\frac{\partial u}{\partial t}+\frac{\partial F(x, t, u)}{\partial x}=v \frac{\partial}{\partial x}\left[G(u) \frac{\partial u}{\partial x}\right]+S(x, t, u)
$$

\footnotetext{
${ }^{*}$ Corresponding author. Fax: +351-22-508-1449.

E-mail address: mmalves@fe.up.pt (M.A. Alves).
} 
with initial values $u(t=0, x)=u_{0}(x)$. The main focus will be, however, in the particular but more difficult hyperbolic equation case that occurs when Eq. (1) is dominated by advection (i.e. diffusivity, $v \rightarrow 0$ ).

It is well known that non-linear hyperbolic equations may develop singularities even for smooth initial conditions, and conventional higher-order discretization schemes applied to the advective term may not be adequate, leading to the appearance of non-physical oscillations. The upwind differencing scheme (UDS1) proposed by Courant et al. [3] is unconditionally stable, but due to its first-order accuracy is not recommended.

To overcome these difficulties an extensive amount of research in computational fluid dynamics has been directed towards the development of accurate and bounded non-linear convective schemes. Several discretization schemes were proposed on the total variation diminishing framework (TVD) [10,27], and more recently on the normalized variable formulation (NVF) [20] and its extension, the normalized variable and space formulation (NVSF) of Darwish and Moukalled [4], to be described below.

In order to improve the computational efficiency, in this work we propose the use of high-resolution schemes combined with an adaptive mesh technique inspired on wavelet theory. In this approach, higherorder accuracy is combined with a multiresolution mesh refinement technique in order to achieve a nearly constant discretization error throughout the computational domain, and thus reducing the memory and CPU requirements, while keeping the same level of accuracy with respect to the equivalent uniform mesh approach.

The basic idea of multiresolution analysis was formalized in a rigorous way by Mallat [22] in the context of orthonormal wavelets. More recently, Harten [12] proposed a general framework for mutiresolution representation of data in order to reduce the number of flux computations with expensive methods such as ENO schemes [11]. In Harten's approach the ENO scheme is used near singularities, while on the smooth regions the numerical fluxes are evaluated with inexpensive interpolations from surrounding grid points. This methodology may produce moderate CPU reductions, but is rather limited since for each time step the solution is still represented on the finest grid. In the approach adopted in this work, the multiresolution representation of data is used to adequately adapt the mesh. In regions of strong gradients a higher number of grid points is allocated while on the smooth regions only the low grid resolution levels are used, without loss of any relevant information. The rejected grid points can easily be reconstructed from lower grid resolution levels, if necessary. This fully adaptive multiresolution approach is similar to the wavelet-based grid generation method proposed by Jameson [15] for orthogonal Daubechies wavelets and the formulation of Bertoluzza [1] and Holmström [14] for interpolating wavelets.

The main advantage of the dynamical adaptation of the mesh based on the multiresolution approach lies in the fact that, frequently, only very small parts of the domain exhibit sharp gradients. In these situations, it is a much better alternative to store only the significant information and perform the time integration in this reduced set of grid points. There is, however, the need to dynamically adapt the mesh as the solution evolves but, as will be demonstrated, this overhead is not significant and globally there are significant memory and CPU time savings as compared to the corresponding fixed grid approach.

This paper is organized as follows: the SMART high-resolution scheme is presented in the context of the NVSF. Then, the multiresolution adaptive strategy is explained and applied to the solution of five test cases which include hyperbolic PDEs with very strong gradients and the classical shock-tube problem of gas dynamics. The paper ends with a summary of the main conclusions.

\section{High-resolution schemes}

Discretization of the convection term in Eq. (1) deserves special treatment, as described in Section 1. For the implementation of high-resolution schemes we follow the NVSF of Darwish and Moukalled [4], which 
is an extension to non-uniform grids of the NVF of Leonard [20,21]. In the NVSF approach, the convection flux $F$ and the co-ordinate $x$ are normalized as

$$
\begin{aligned}
& \widehat{F}=\frac{F-F_{\mathrm{U}}}{F_{\mathrm{D}}-F_{\mathrm{U}}}, \\
& \widehat{x}=\frac{x-x_{\mathrm{U}}}{x_{\mathrm{D}}-x_{\mathrm{U}}},
\end{aligned}
$$

where the subscripts $U$ and $\mathrm{D}$ refer to the upstream and downstream points to node $\mathrm{P}$ which is, itself, upstream of the face $\mathrm{f}$ under consideration, as shown in Fig. 1. Note that the labeling of the grid points depends on the local velocity, $a$, calculated at face $\mathrm{f}$ by linear interpolation from surrounding nodes:

$$
a_{\mathrm{f}=i+1 / 2}=a_{i}+\frac{x_{\mathrm{f}}-x_{i}}{x_{i+1}-x_{i}}\left(a_{i+1}-a_{i}\right)
$$

with $a_{i} \equiv(\mathrm{d} F / \mathrm{d} u)_{i}$.

In general, a differencing scheme (of order 3 or less) is used to evaluate the face flux $F_{\mathrm{f}}$ as function of the neighbouring cell values ( $\mathrm{U}, \mathrm{P}$ and $\mathrm{D})$, which can be written in the form

$$
F_{\mathrm{f}}=f\left(F_{\mathrm{U}}, F_{\mathrm{P}}, F_{\mathrm{D}}, x_{\mathrm{U}}, x_{\mathrm{P}}, x_{\mathrm{D}}, x_{\mathrm{f}}\right) .
$$

Using the NVSF, Eq. (5) can be rewritten as

$$
\widehat{F}_{\mathrm{f}}=f\left(\widehat{F}_{\mathrm{P}}, \widehat{x}_{\mathrm{P}}, \widehat{x}_{\mathrm{f}}\right),
$$

where

$$
\begin{gathered}
\widehat{F}_{\mathrm{P}}=\frac{F_{\mathrm{P}}-F_{\mathrm{U}}}{F_{\mathrm{D}}-F_{\mathrm{U}}}, \\
\widehat{F}_{\mathrm{f}}=\frac{F_{\mathrm{f}}-F_{\mathrm{U}}}{F_{\mathrm{D}}-F_{\mathrm{U}}}, \\
\widehat{x}_{\mathrm{P}}=\frac{x_{\mathrm{P}}-x_{\mathrm{U}}}{x_{\mathrm{D}}-x_{\mathrm{U}}}, \\
\widehat{x}_{\mathrm{f}}=\frac{x_{\mathrm{f}}-x_{\mathrm{U}}}{x_{\mathrm{D}}-x_{\mathrm{U}}} .
\end{gathered}
$$

The appearance of unphysical oscillations in the computed solution can be avoided if the face flux $F_{\mathrm{f}}$ lies within the bounds of its surrounding values, $F_{\mathrm{P}}$ and $F_{\mathrm{D}}$. Gaskell and Lau [8] formulated the convective boundedness criterion ( $\mathrm{CBC}$ ), which is an elegant and rigorous framework for the development of highresolution schemes. The $\mathrm{CBC}$ states that the functional relationship of an interpolation scheme applied to a generic face f, $\widehat{F}_{\mathrm{f}}=\Omega\left(\widehat{F}_{\mathrm{P}}\right)$, must be continuous and bounded from below by $\widehat{F}_{\mathrm{f}}=\widehat{F}_{\mathrm{P}}$ and from above by unity, in the monotonic range $0<\widehat{F}_{\mathrm{P}}<1$. In the non-monotonic ranges $\left(\widehat{F}_{\mathrm{P}} \leqslant 0\right.$ and $\left.\widehat{F}_{\mathrm{P}} \geqslant 1\right)$ the non-linear

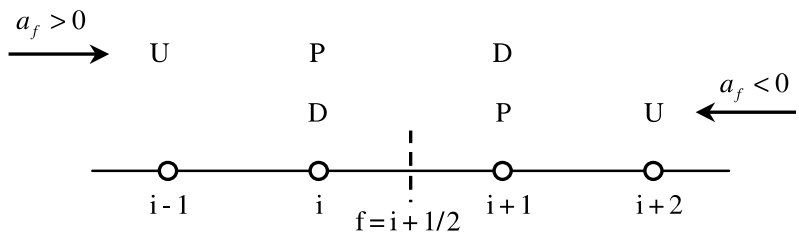

Fig. 1. Definition of local variables. 


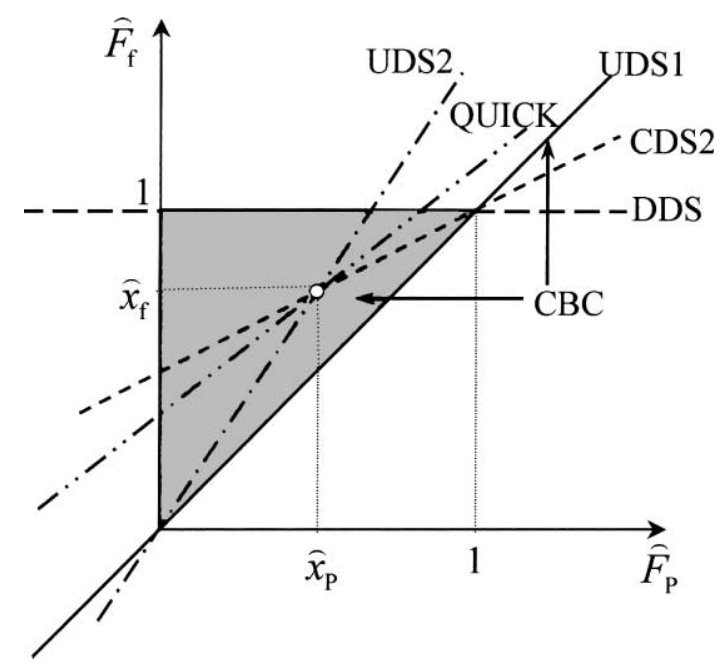

Fig. 2. NVD: illustration of the $\mathrm{CBC}$ and representation of some linear differencing schemes.

high-resolution scheme must revert to the first-order upwind (UDS1) in order to preserve boundedness, i.e. $F_{\mathrm{f}}=F_{\mathrm{P}}$. The CBC is easily illustrated on a normalized variable diagram (NVD), as shown in Fig. 2 by the shadowed area together with the line of unitary slope. The straight lines shown in this figure are for the firstorder upwind (UDS1) and downwind differencing schemes (DDS), the second-order upwind (UDS2) [24] and central differences schemes (CDS2) and for the third-order quadratic upwind scheme (QUICK or UDS3) of Leonard [19]. The only linear differencing scheme that fully satisfies the CBC is the first-order UDS1. Indeed, in a important paper, Godunov [9] showed that all monotone linear schemes achieve, at most, first-order accuracy. Hence, the only way to circumvent this limitation is by introducing non-linear composite schemes, and the CBC together with the NVD provide an easy framework to create high-resolution schemes.

A number of high-resolution schemes were formulated in the context of the NVSF methodology. In this work we selected the popular MINMOD [10] and SMART [8] schemes, which are expressed in the NVSF as [4]:

MINMOD: $\widehat{F}_{\mathrm{f}}=\left\{\begin{array}{lll}\frac{\widehat{x}_{\mathrm{f}}}{\widehat{x}_{\mathrm{P}}}, & 0<\widehat{F}_{\mathrm{P}}<\widehat{x}_{\mathrm{P}} & (\mathrm{UDS} 2), \\ \frac{1-\widehat{x}_{\mathrm{f}}}{1-\widehat{x}_{\mathrm{P}}+\frac{\widehat{x}_{\mathrm{f}}-\widehat{x}_{\mathrm{P}}}{1-\widehat{x}_{\mathrm{P}}},} & \widehat{x}_{\mathrm{P}} \leqslant \widehat{F}_{\mathrm{P}}<1 & (\mathrm{CDS} 2), \\ \widehat{F}_{\mathrm{P}}, & \text { elsewhere } & (\mathrm{UDS} 1),\end{array}\right.$

SMART: $\widehat{F}_{\mathrm{f}}=\left\{\begin{array}{lll}\frac{\widehat{x}_{\mathrm{f}}\left(1-3 \widehat{x}_{\mathrm{P}}+2 \widehat{x}_{\mathrm{f}}\right)}{\widehat{x}_{\mathrm{P}}\left(1-\widehat{x}_{\mathrm{P}}\right)} \widehat{F}_{\mathrm{P}}, & 0<\widehat{F}_{\mathrm{P}}<\frac{\widehat{x}_{\mathrm{P}}}{3}, \\ \frac{\widehat{x}_{\mathrm{f}}\left(1-\widehat{x}_{\mathrm{f}}\right)}{\widehat{x}_{\mathrm{P}}\left(1-\widehat{x}_{\mathrm{P}}\right)} \widehat{F}_{\mathrm{P}}+\frac{\widehat{x}_{\mathrm{f}}\left(\widehat{x}_{\mathrm{f}}-\widehat{x}_{\mathrm{P}}\right)}{1-\widehat{x}_{\mathrm{P}}}, & \frac{\widehat{x}_{\mathrm{P}}}{3} \leqslant \widehat{F}_{\mathrm{P}} \leqslant \frac{\widehat{x}_{\mathrm{P}}}{\widehat{x}_{\mathrm{f}}}\left(1+\widehat{x}_{\mathrm{f}}-\widehat{x}_{\mathrm{P}}\right) & (\mathrm{QUICK}), \\ 1, & \frac{\widehat{x}_{\mathrm{P}}}{\widehat{x}_{\mathrm{f}}}\left(1+\widehat{x}_{\mathrm{f}}-\widehat{x}_{\mathrm{P}}\right)<\widehat{F}_{\mathrm{P}}<1 & (\text { DDS }), \\ \widehat{F}_{\mathrm{P}}, & \text { elsewhere } & \text { (UDS1), }\end{array}\right.$ 


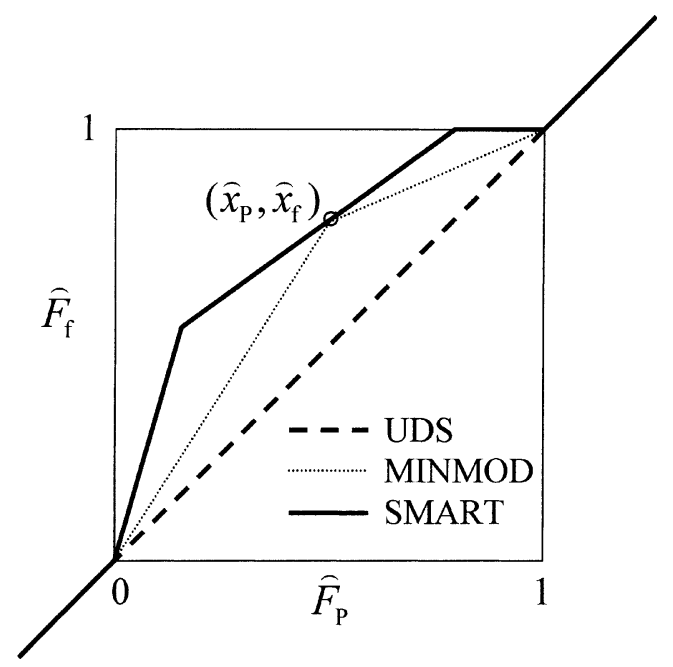

Fig. 3. NVD representation of the MINMOD and SMART high-resolution schemes.

and are illustrated in Fig. 3 in the NVD. It should be noted that both high-resolution schemes cross the point $\left(\widehat{x}_{\mathrm{P}}, \widehat{x}_{\mathrm{f}}\right)$ which is a requirement for second-order accuracy [4].

The non-linear Eqs. (11) and (12) can be written in compact form as

$$
\begin{aligned}
& \widehat{F}_{\mathrm{f}}(\mathrm{MINMOD})=\max \left[\widehat{F}_{\mathrm{P}}, \min \left(\frac{\widehat{x}_{\mathrm{f}}}{\widehat{x}_{\mathrm{P}}} \widehat{F}_{\mathrm{P}}, \frac{1-\widehat{x}_{\mathrm{f}}}{1-\widehat{x}_{\mathrm{P}}} \widehat{F}_{\mathrm{P}}+\frac{\widehat{x}_{\mathrm{f}}-\widehat{x}_{\mathrm{P}}}{1-\widehat{x}_{\mathrm{P}}}\right)\right], \\
& \widehat{F}_{\mathrm{f}}(\mathrm{SMART})=\max \left[\widehat{F}_{\mathrm{P}}, \min \left(\frac{\widehat{x}_{\mathrm{f}}\left(1-3 \widehat{x}_{\mathrm{P}}+2 \widehat{x}_{\mathrm{f}}\right)}{\widehat{x}_{\mathrm{P}}\left(1-\widehat{x}_{\mathrm{P}}\right)} \widehat{F}_{\mathrm{P}}, \frac{\widehat{x}_{\mathrm{f}}\left(1-\widehat{x}_{\mathrm{f}}\right)}{\widehat{x}_{\mathrm{P}}\left(1-\widehat{x}_{\mathrm{P}}\right)} \widehat{F}_{\mathrm{P}}+\frac{\widehat{x}_{\mathrm{f}}\left(\widehat{x}_{\mathrm{f}}-\widehat{x}_{\mathrm{P}}\right)}{1-\widehat{x}_{\mathrm{P}}}, 1\right)\right]
\end{aligned}
$$

which are better suitable for implementation in a numerical code.

Calculation of a face flux is a straightforward task, and takes the following four steps:

(i) calculate the face velocity using Eq. (4) and identify the P, U and D grid points (as illustrated in Fig. 1);

(ii) calculate the normalized values of $\widehat{F}_{\mathrm{P}}, \widehat{x}_{\mathrm{P}}$ and $\widehat{x}_{\mathrm{f}}$ using Eqs. (7), (9) and (10);

(iii) choose a high-resolution scheme and compute the normalized face flux $\widehat{F}_{\mathrm{f}}$ using Eq. (13) or (14);

(iv) revert the face flux back to dimensional form:

$$
F_{\mathrm{f}}=F_{\mathrm{U}}+\widehat{F}_{\mathrm{f}}\left(F_{\mathrm{D}}-F_{\mathrm{U}}\right) \text {. }
$$

\subsection{Multiresolution representation of data}

Consider a set of dyadic grids on the form

$$
V^{j}=\left\{x_{k}^{j} \in \mathbb{R}: x_{k}^{j}=2^{-j} k, k \in \mathbb{Z}\right\}, \quad j \in \mathbb{Z}
$$

where $j$ identifies the resolution level and $k$ the spatial location, as illustrated in Fig. 4. Assume that the solution is known on grid $V^{j}$, and we want to extend it to the finer grid $V^{j+1}$. Values on the even-numbered grid points are known from the corresponding values on the lower resolution grid:

$$
u_{2 k}^{j+1}=u_{k}^{j} \text {. }
$$




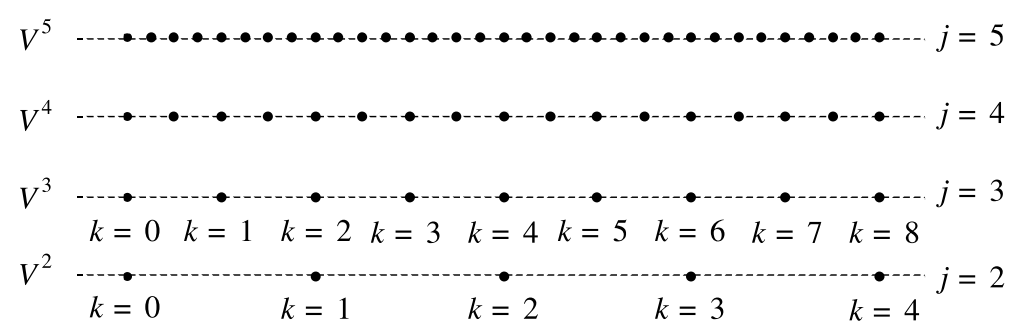

Fig. 4. Example of points in a dyadic grid.

Values on the odd-numbered grid points in $V^{j+1}$ are computed by adequate interpolation from the known even-numbered grid points (present in $V^{j}$ ). A common practice in wavelet-based methods is to utilize symmetric interpolating polynomials [14,28], but, to be consistent with the discretization used for the problematic convection term, we propose the use of the high-resolution interpolating schemes. The normalized difference between the interpolated value, $I^{j}\left(u_{2 k+1}^{j+1}\right)$, and the real one, $u_{2 k+1}^{j+1}$, is called interpolative error coefficient, $d_{k}^{j}$, and is expressed as

$$
d_{k}^{j}=\left|u_{2 k+1}^{j+1}-I^{j}\left(u_{2 k+1}^{j+1}\right)\right| / u_{\mathrm{ref}},
$$

where $u_{\text {ref }}$ is a reference value of the dependent variable. We suggest the use of either $u_{\text {ref }}=\max \left(\left|u_{2 i+1}^{j+1}\right|\right.$, $i=0, \ldots, 2^{j}$ ) or a known maximum value of the dependent variable.

The interpolative error coefficients are thus a measure of the local "irregular" behaviour of the function. If the value of $d_{k}^{j}$ is below a given (small) threshold $\varepsilon$, then the grid point $x_{2 k+1}^{j+1}$ can be rejected without loss of significant information (for small $\varepsilon$ ) since it can be reconstructed from the preserved information obtained from the coarser grid $V^{j}$. A function that varies abruptly only in a narrow area of the domain will have most of the $d_{k}^{j}$ coefficients close to zero and so the information can be compressed with great efficiency without loss of accuracy. This multiresolution approach has been also extensively studied in the context of image compression, and the interested reader is referred to the work of Zhou [29].

Finally, it should be noted that the multiresolution approach must select the relevant grid points in a tree-like structure, as illustrated in Fig. 5. It is necessary to guarantee that if the point $x_{2 k+1}^{j+1}$ is retained in the adaptation algorithm, then the function values at the locations $x_{k-1}^{j}, x_{k}^{j}, x_{k+1}^{j}$ and $x_{k+2}^{j}$ must be preserved, in order to enable the reconstruction of the $u_{2 k+1}^{j+1}$ value in the next adaptation step, and also to evaluate $d_{k}^{j}$ (as shown in Fig. 5). The maximum level of resolution is specified by the user so that grid coalescence is avoided in problematic regions (typically in this work we used $J_{\max }=12$ ). The user also supplies the minimum level of resolution, and all the grid points pertaining to this level of resolution are always conserved throughout the computations (typically in this work we set $J_{\min }=4$ ).

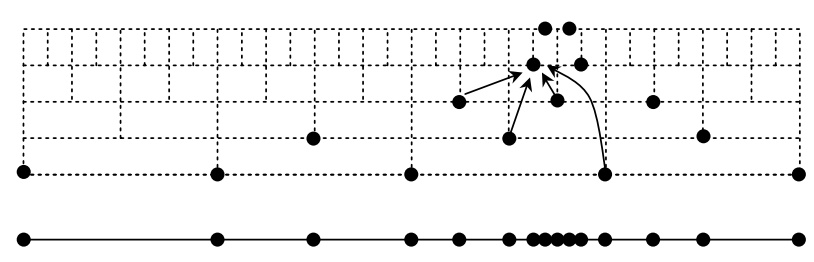

Fig. 5. Example of a typical grid with tree structure obtained with the multiresolution adaptation strategy. 


\section{Adaptation strategy}

During the time integration of the PDE the grid should be continuously adapted, so that it can automatically adjust to reflect the transient behaviour of the solution. The grid adaptation strategy can be summarized in the following steps:

(i) Given discrete function values $u_{k}^{j}$, in the grid $V^{j}$ at time $t=t_{1}$, compute the interpolative error coefficients $d_{k}^{j}$ for $J_{\min } \leqslant j \leqslant J_{\max }-1$.

(ii) Identify the coefficients $d_{k}^{j}$ that are above the predefined threshold $\varepsilon$. The corresponding grid points, $x_{2 k+1}^{j+1}$, are included in an indicator.

(iii) Add points $x_{2(k+i)+1}^{j+1}, i=-N_{\mathrm{L}}, N_{\mathrm{R}}$ to the indicator. These are the grid points to the right, $N_{\mathrm{R}}$, and to the left, $N_{\mathrm{L}}$, of the previous ones, at the same resolution level. This operation is done in order to account for possible displacement of the sharp features of the solution during the next time integration steps, thus reducing the frequency of mesh adaptations. The maximum time allowed between mesh adaptations (a conservative value) is given by

$$
\Delta t_{\text {adapt }}=\max \left(-\frac{N_{\mathrm{L}} \Delta x_{\min }}{a_{\min }}, \frac{N_{\mathrm{R}} \Delta x_{\min }}{a_{\max }}\right)
$$

in order to avoid that the sharp front leaves the maximum resolution level leading to a loss of precision.

(iv) Add points $x_{4 k+1}^{j+2}$ and $x_{4 k+3}^{j+2}$ to the indicator. These are the grid points in the resolution level immediately above. This step accounts for the possibility of the solution becoming "steeper" in this region.

(v) Add to the indicator the grid points associated to the lower resolution level, $J_{\min }$. These are the "basic" grid points, which are always present.

(vi) Begin at resolution level $j=J_{\max }-1$, and recursively extend the indicator, so that all the grid points necessary for the calculation of the existing $j$ th level $u_{k}^{j}$ coefficients are included.

\subsection{Temporal integration}

Time integration was done with the public domain first-order ordinary differential equation (ODE) solver LSODA [23]. This flexible routine solves the initial boundary problem for stiff or non-stiff systems of first-order ODEs by dynamically switching the integration methods in accordance to the stiffness of the problem. For non-stiff systems it uses Adams method with variable step size and order (up to 12th order), while for stiff systems it uses Gear (or BDF) method with variable step size and order (up to fifth order). The admissible error used in the time integration was always small enough in order to assure that the differences between the exact and computed values are mainly due to inaccuracies in the space discretization.

The complete algorithm, including grid adaptation strategy and temporal integration, is summarized in Fig. 6.

\section{Results}

In this section we present the results obtained in five test cases to assess the accuracy and robustness of the proposed strategy under different situations. We begin by solving the classical linear advection equation. Then we solve Burgers' non-linear equation with a smooth initial condition, to show the ability of the method to capture a self-sharpening front. The inclusion of a strong source term is illustrated in the third example and then we proceed to the solution of a simple system of two hyperbolic PDEs which are linked by source terms. In the final example we solve the more complex system of Euler equations for gas dynamics. 


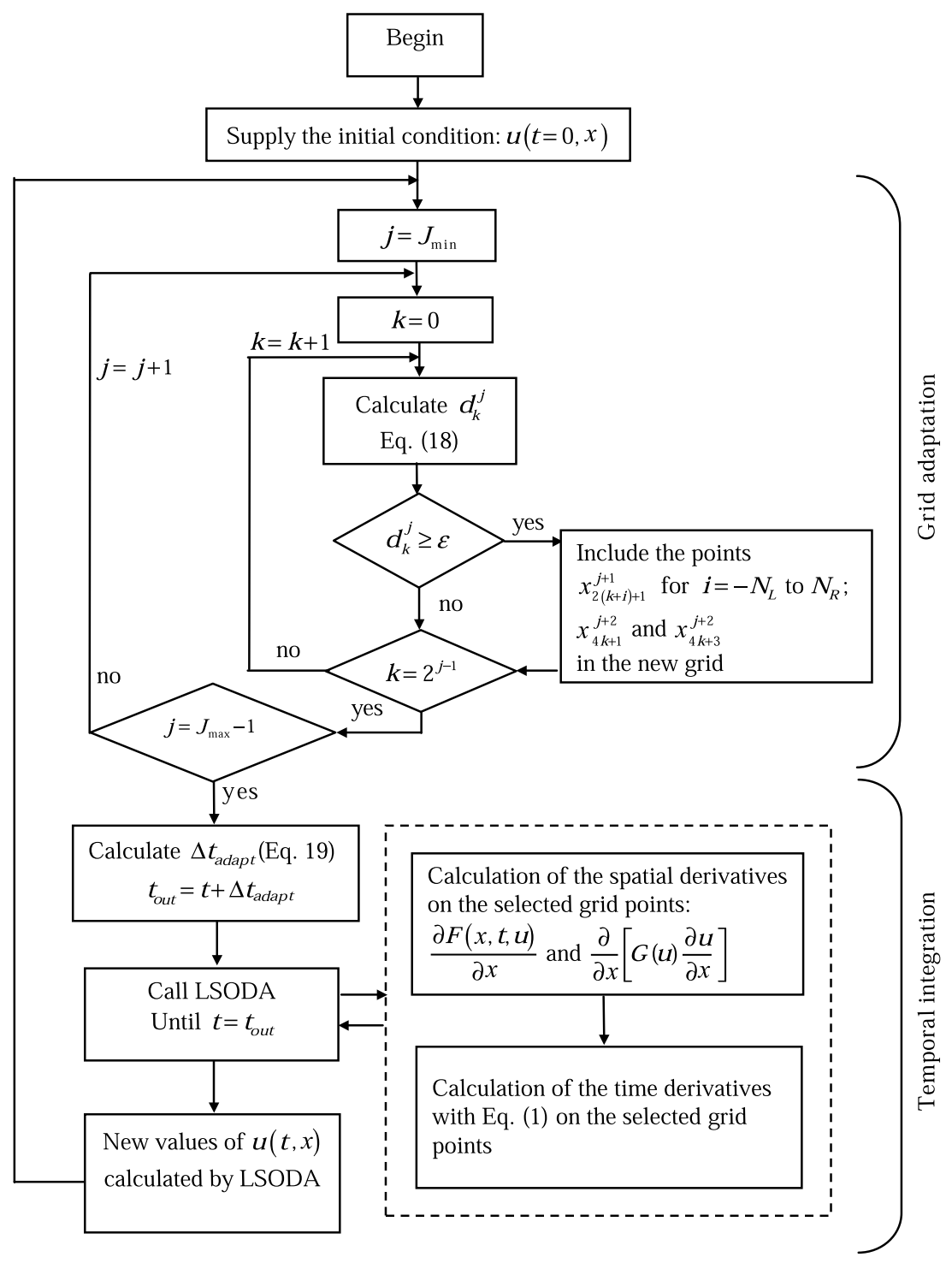

Fig. 6. Flow chart describing the proposed algorithm.

All the calculations were performed in a $700 \mathrm{MHz}$ Intel Pentium III ${ }^{\circledR}$ personal computer with $128 \mathrm{MB}$ DRAM.

\subsection{Linear advection equation}

The linear advection equation,

$$
\frac{\partial u}{\partial t}=-a \frac{\partial u}{\partial x}
$$


is used to assess the robustness and accuracy of the discretization schemes. For convenience, $a=1$ was used. The initial and boundary conditions used were the classical benchmark advection of a step profile, given by

$$
u(x, 0)=1, \quad u(0, t)=0 .
$$

The numerical results obtained in two uniform meshes with $2^{8}+1$ and $2^{10}+1$ grid points are presented in Fig. 7 to illustrate convergence with mesh refinement for this difficult problem. As expected, the unbounded schemes show severe unphysical oscillations, which decrease in magnitude and increase in frequency with mesh refinement. On the other hand, the first-order upwind and the high-resolution schemes do not suffer from this problem due to their inherent boundedness property. However, due to the low order of accuracy, the first-order upwind scheme is not recommended, since it induces large diffusive errors in the computed solution. Indeed, it is nowadays common policy for most archival journals that first-order schemes should be avoided [7]. The SMART scheme was found to be highly robust and clearly the most accurate, thus making it the chosen scheme to the treatment of the convection term in the following examples, unless otherwise stated.

The present test case, with an exact solution given by a moving step with velocity $a=1$, offers a convenient means of assessing the performance of the proposed adaptation strategy. Guided by the previous results, the convection term was discretized with the SMART high-resolution scheme in the adapted nonuniform grid. Furthermore, it should be clear that there is no need to reconstruct any values from lower levels of resolution (a very time-consuming process), except during the grid adaptation stage of the algorithm.

The number of grid points used by the adaptation algorithm for two threshold values $\left(\varepsilon=10^{-3}\right.$ and $10^{-5}$ ) is presented in Fig. 8. The minimum and maximum levels of resolution adopted were $J_{\min }=4$ and $J_{\max }=12$, respectively, and the parameters used in the adaptation algorithm were $N_{\mathrm{R}}=N_{\mathrm{L}}=2$.

The computed solution with the SMART scheme is presented in Fig. 9(a) at $t=0.5$. As can be seen, the adaptation algorithm is performing well and the step is predicted with high accuracy. The distribution of the grid points is represented in Fig. 9(b) in terms of position and resolution level. The smooth regions of the solution are resolved by the coarse resolution levels while the higher levels are only required near the step location, thus illustrating the efficient data compression of the adaptive strategy.

The same problem was solved on equivalent uniform meshes having $2^{J_{\max }}+1$ grid points, with the aim of calculating the speed-up factors, defined as the ratio of CPU times for the uniform mesh and the adaptive calculations. The accuracy of the computed solutions is equivalent for both approaches for each $J_{\max }$ but, as seen in Table 1, the speed-up factors are high, thus proving the efficiency of the proposed multiresolution approach. In addition, the speed-up factor is seen to increase significantly with mesh refinement (almost at the optimum rate of 2), and can be as high as we want, which contrasts with the multiresolution approach of Harten [12] where the attained speed-up factors are only of order 2-5.

\subsection{Burgers' equation}

This example considers the numerical solution of the classical inviscid Burgers' equation [6]:

$$
\frac{\partial u}{\partial t}+\frac{\partial\left(u^{2} / 2\right)}{\partial x}=0
$$

where $u, x$ and $t$ are the dimensionless dependent and independent variables which can be seen as velocity (or other advected property), space co-ordinate and time, respectively.

Burgers' equation is similar in form to the linear advection equation, except that the convective term is non-linear thus allowing discontinuous solutions to develop. The initial and boundary conditions considered for this standard test were the same as in [2]: 

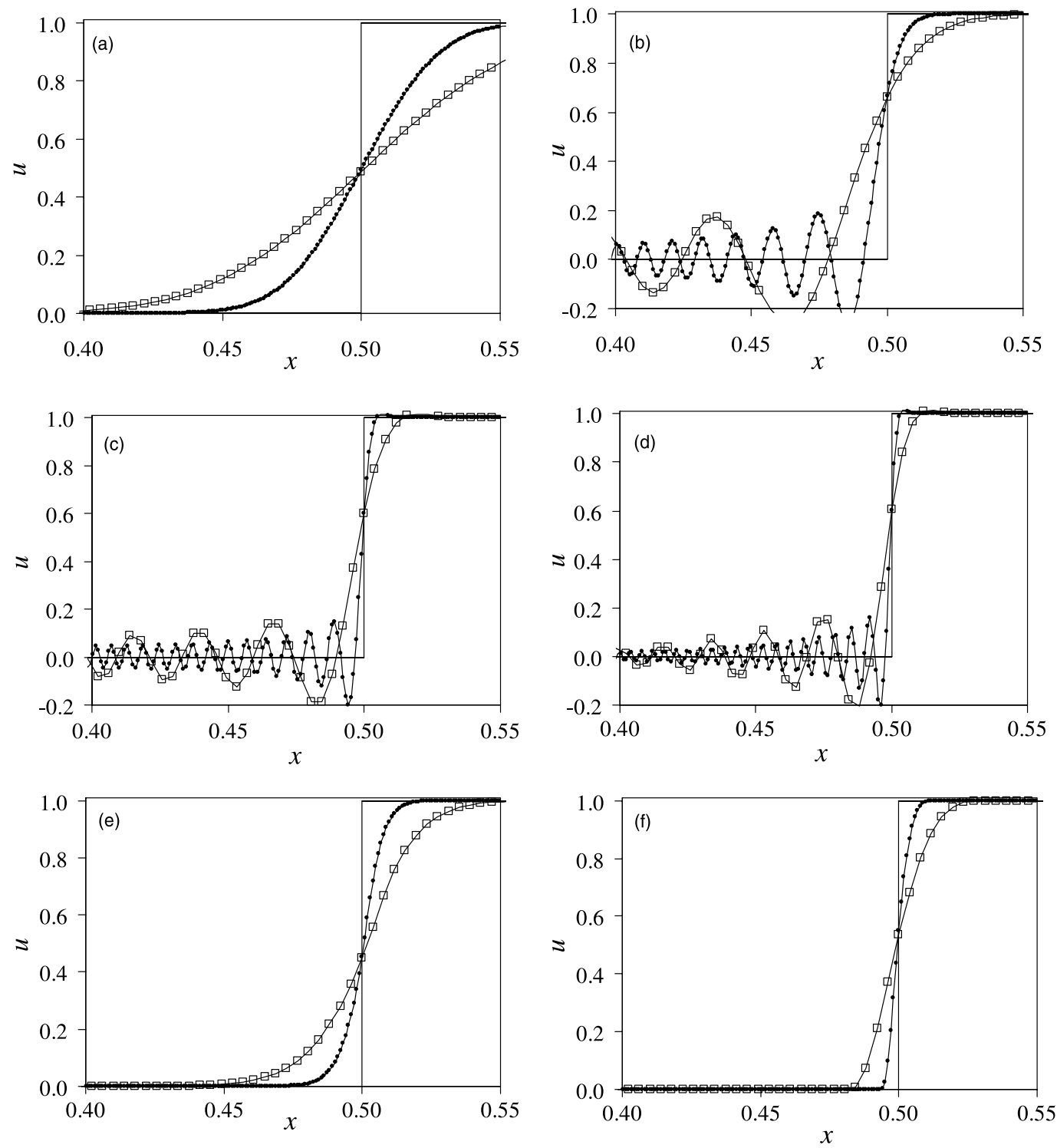

Fig. 7. Effect of mesh refinement in the solution of the linear advection equation with a step profile. Results for $t=0.5$ obtained in two uniform meshes with $2^{J}+1$ grid points $(-\square-J=8,-\mathbf{-}-J=10$, - Theoretical): (a) UDS1; (b) CDS2; (c) CDS4; (d) cubic splines; (e) MINMOD; and (f) SMART.

$$
u(x, 0)=\sin (2 \pi x)+\sin (\pi x) / 2, \quad u(0, t)=u(1, t)=0 .
$$

This smooth initial condition is very convenient to test the ability of the adaptation method for adequately capturing the strong shock that is formed at $t \cong 0.158$, and then propagates to the right. The computed results and the exact solution are presented in Fig. 10 for various elapsed times, showing a perfect agreement and tight shock resolution. The parameters used in the adaptation algorithm were $\varepsilon=10^{-3}$, $N_{\mathrm{R}}=N_{\mathrm{L}}=2$ and minimum and maximum resolution levels of 4 and 12, respectively. Fig. 10 also shows the 


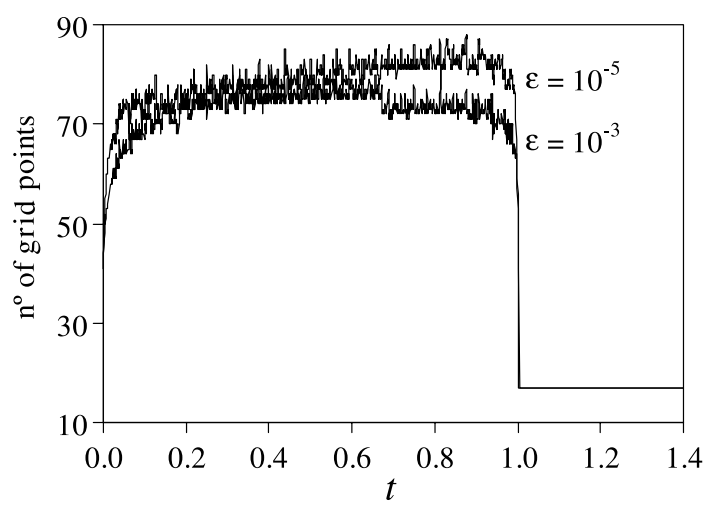

Fig. 8. Number of grid points used by the adaptation algorithm for the solution of the linear advection equation with a step profile, for two threshold values, $\varepsilon=10^{-3}$ and $10^{-5}$.
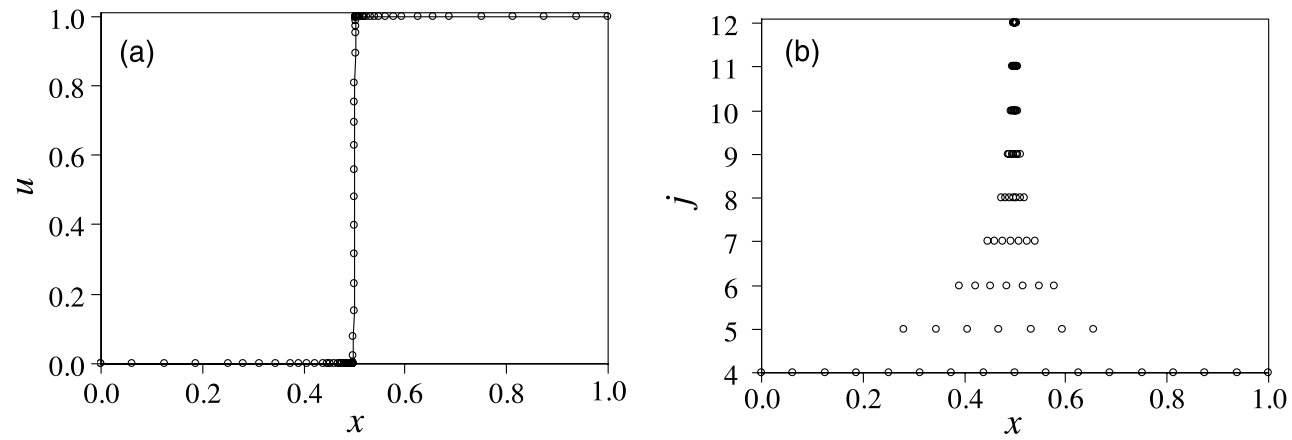

Fig. 9. (a) Solution of the linear advection equation with a step profile at $t=0.5$ and (b) corresponding distribution of the grid points in terms of spatial location and resolution level. The parameters used in the adaptation algorithm were $\varepsilon=10^{-3}, N_{\mathrm{R}}=N_{\mathrm{L}}=2, J_{\min }=4$ and $J_{\max }=12$.

Table 1

Speed-up factors and CPU times for advective transport of a profile $(t=0.5)$

\begin{tabular}{|c|c|c|c|c|c|}
\hline \multirow[t]{2}{*}{$J_{\max }$} & \multicolumn{2}{|c|}{ Uniform mesh } & \multicolumn{3}{|c|}{ Adaptive } \\
\hline & $\mathrm{NGP}^{\mathrm{a}}$ & CPU (s) & $\mathrm{NGP}^{\mathrm{a}}$ & CPU (s) & Speed-up \\
\hline 8 & 257 & 3.30 & 49 & 0.66 & 5.0 \\
\hline 9 & 513 & 12.8 & 58 & 1.76 & 7.3 \\
\hline 10 & 1025 & 48.9 & 66 & 3.79 & 12.9 \\
\hline 11 & 2049 & 222.2 & 76 & 8.52 & 26.1 \\
\hline 12 & 4097 & 1006.2 & 82 & 19.5 & 51.6 \\
\hline
\end{tabular}

${ }^{\mathrm{a}}$ Number of grid points. For the adaptive strategy indicates the number of points at $t=0.5$.

location and the resolution levels of the grid points in the adaptive mesh at $t=0, t=0.158$ and $t=1$. At this last time, the grid points are clearly concentrated and distributed through the various resolution levels at the exact position of the shock. 

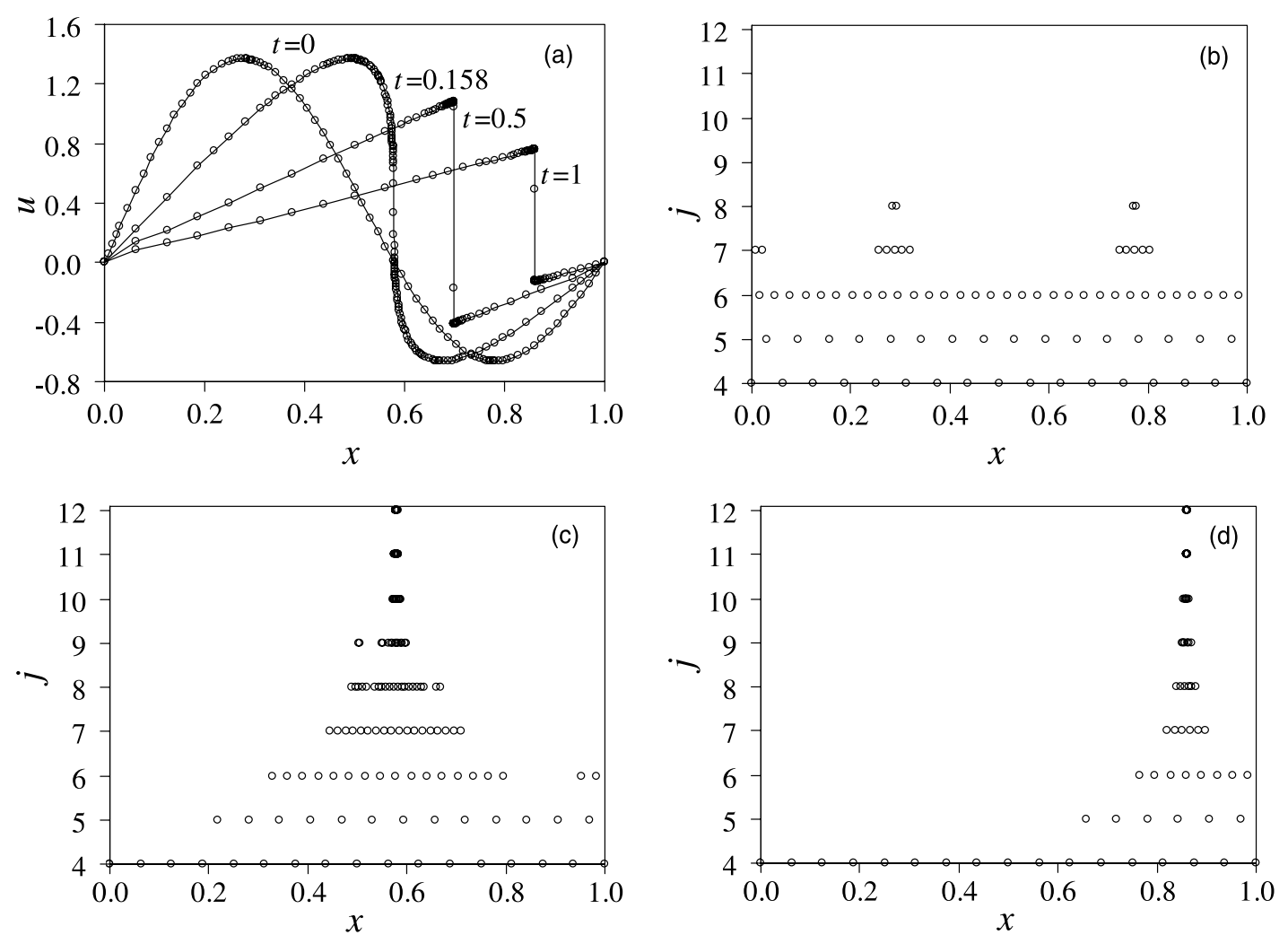

Fig. 10. (a) Solution of Burgers' equation for different $t$ values. Distribution of the grid points in terms of spatial location and resolution level for (b) $t=0$, (c) $t=0.158$, and (d) $t=1$, respectively. The parameters used in the adaptation algorithm are $\varepsilon=10^{-3}$, $N_{\mathrm{R}}=N_{\mathrm{L}}=2, J_{\min }=4$ and $J_{\max }=12$.

Fig. 11 shows the time evolution of the number of grid points used by the adaptation algorithm. It is interesting to note that the appearance of the discontinuity in the computed solution corresponds to a significant decrease in the number of grid points needed to compute the solution to the prescribed

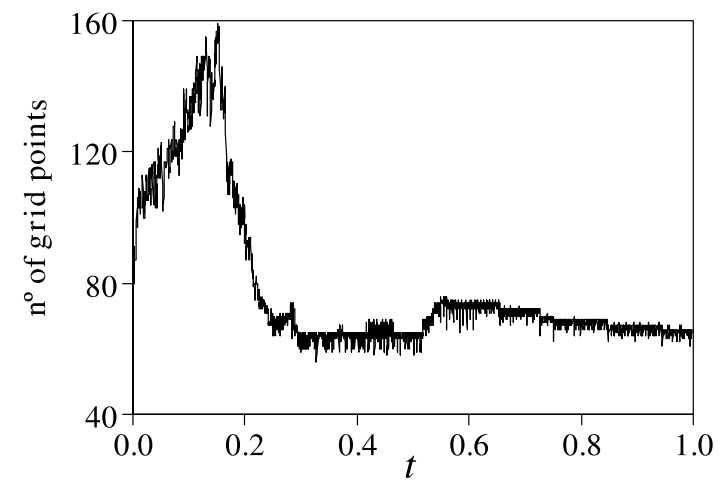

Fig. 11. Time evolution of the number of grid points used by the adaptation algorithm for the solution of Burgers' equation. Adaptation parameters as in Fig. 10. 
Table 2

Speed-up factors and CPU times for Burgers' equation $(t=1)$

\begin{tabular}{|c|c|c|c|c|c|}
\hline \multirow[t]{2}{*}{$J_{\max }$} & \multicolumn{2}{|c|}{ Uniform mesh } & \multicolumn{3}{|c|}{ Adaptive } \\
\hline & $\mathrm{NGP}^{\mathrm{a}}$ & CPU (s) & $\mathrm{NGP}^{\mathrm{a}}$ & CPU (s) & Speed-up \\
\hline 8 & 257 & 8.56 & 62 & 3.35 & 2.6 \\
\hline 9 & 513 & 32.5 & 51 & 5.10 & 6.4 \\
\hline 10 & 1025 & 130.4 & 55 & 10.3 & 12.7 \\
\hline 11 & 2049 & 553.1 & 59 & 21.4 & 25.8 \\
\hline 12 & 4097 & 2433 & 64 & 48.5 & 50.2 \\
\hline
\end{tabular}

${ }^{a}$ Number of grid points. For the adaptive strategy indicates the number of points at $t=1$.

threshold. This result is very important since it demonstrates a virtue of the current strategy as compared to other approaches, such as the moving mesh method, where a constant number of grid points are used throughout the computations. The proposed strategy uses only the grid points that are actually necessary to attain a given precision, and so is more versatile and efficient.

Table 2 compares the CPU times required by the constant grid and adaptive approaches. Once again the speed-up factors are notorious, which shows the good performance of the proposed adaptive strategy.

\subsection{Glacier growth model}

This example analyses a simple one-dimensional model for glacier growth [16], which is useful to study the possible effect of source terms and non-linear fluxes. Consider a glacier, of height $h(x, t)$, that rests upon a flat mountain. The laws for conservation of mass and momentum govern the flow of the glacier, assumed to be an incompressible viscoplastic fluid. It can be shown that the evolution of the glacier height is described by the following non-homogeneous parabolic convection-diffusion equation (for a detailed derivation consult [16]):

$$
\frac{\partial h}{\partial t}+\frac{\partial F(h)}{\partial x}=v \frac{\partial}{\partial x}\left[G(h) \frac{\partial h}{\partial x}\right]+S(x, t, h) .
$$

Following [16,17] we solved Eq. (22) with $v=0.01, F(h)=\left(h+3 h^{6}\right) / 4$ and $G(h)=3 h^{6}$, in the interval $-0.5 \leqslant x \leqslant 2.5$, subjected to the following initial and boundary conditions:

$$
h(x, 0)=\left\{\begin{array}{ll}
1, & x \leqslant 0, \\
0, & x>0,
\end{array} \quad h(0, t)=1\right.
$$

which describe a simplified model of an outlet into a valley. To complete this simple model we used the source $S(x, t, h)=S_{0}(x)$ if $h(x, t)>0$, and $S(x, t, h)=\max \left\{S_{0}(x), 0\right\}$ if $h(x, t)=0$, where

$$
S_{0}(x)= \begin{cases}0, & x<-0.4, \\ (x+0.4) / 2, & -0.4 \leqslant x \leqslant-0.2, \\ -x / 2, & x>-0.2\end{cases}
$$

which does not account for seasonal variations (i.e. the source term is independent of time).

The solution for time evolution of the glacier height and the distribution of the grid points at $t=1$ are presented in Fig. 12. The number of grid points used by the adaptation algorithm is shown in Fig. 13, and it can be concluded that a steady-state solution is achieved approximately at $t=3.1$, when there is an exact balance between the effects of ablation and of the glacier forward movement.

As an interesting variation of the above problem, we consider now the growth of a newly formed glacier, that is $h(x, 0)=0$. Let the glacier be restricted to the interval $[-5,5]$ and assume that the source term is [16] 

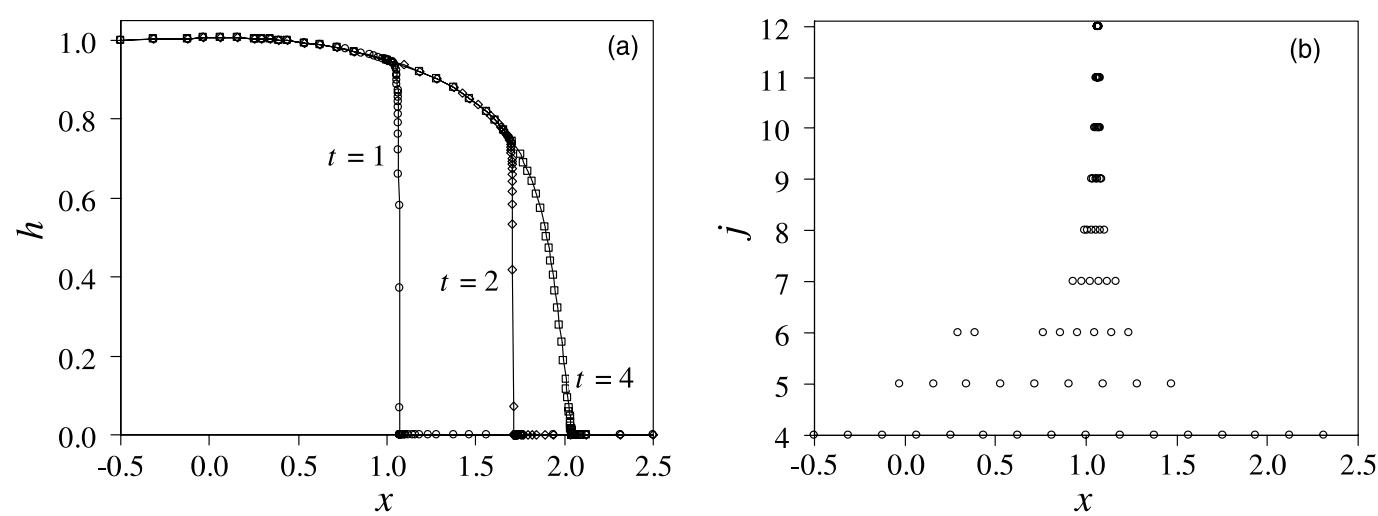

Fig. 12. Solution of the glacier growth model: (a) glacier height at $t=1,2$ and 4; (b) distribution of the grid points in terms of spatial location and resolution level at $t=1$. The parameters used in the adaptation algorithm are $\varepsilon=10^{-3}, N_{\mathrm{R}}=N_{\mathrm{L}}=2, J_{\text {min }}=4$ and $J_{\max }=12$.

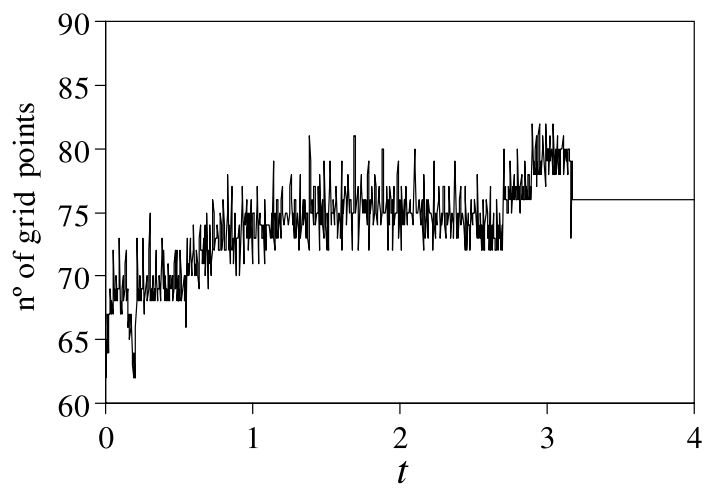

Fig. 13. Number of grid point used by the adaptation algorithm for the solution of the glacier growth model. Adaptation parameters as in Fig. 12.

$$
S(x, t)= \begin{cases}0, & x \leqslant-5 \\ -0.01 x+0.05 \sin (2 \pi t), & x>-5\end{cases}
$$

which now includes seasonal variations (modeled by the time-dependent sine term).

The computed solution representing the time evolution of the glacier growth is given in Fig. 14, which shows profiles of the glacier height at three different times, together with the distribution of the grid points across the resolution levels at the middle time, when a solidification front is present.

The proposed adaptive multiresolution strategy is particularly adequate for this difficult problem because it can adequately capture and resolve the main discontinuity due to the solidification front, but it is also able to resolve other small details of the solution due to the variable source term. The small height oscillations observed on the glacier are originated by seasonal variations (note that the source term, when viewed on the $x-t$ plane, tends to form an interference pattern due to the phase lag of the sine functions at different $x$-positions, which is the direct cause of the height oscillations).

It is instructive to compare the present solution with those of [16,17]; the resolution here is apparently better. In fact in [17] the authors did not believe that the oscillations were of "physical" nature; they ob- 

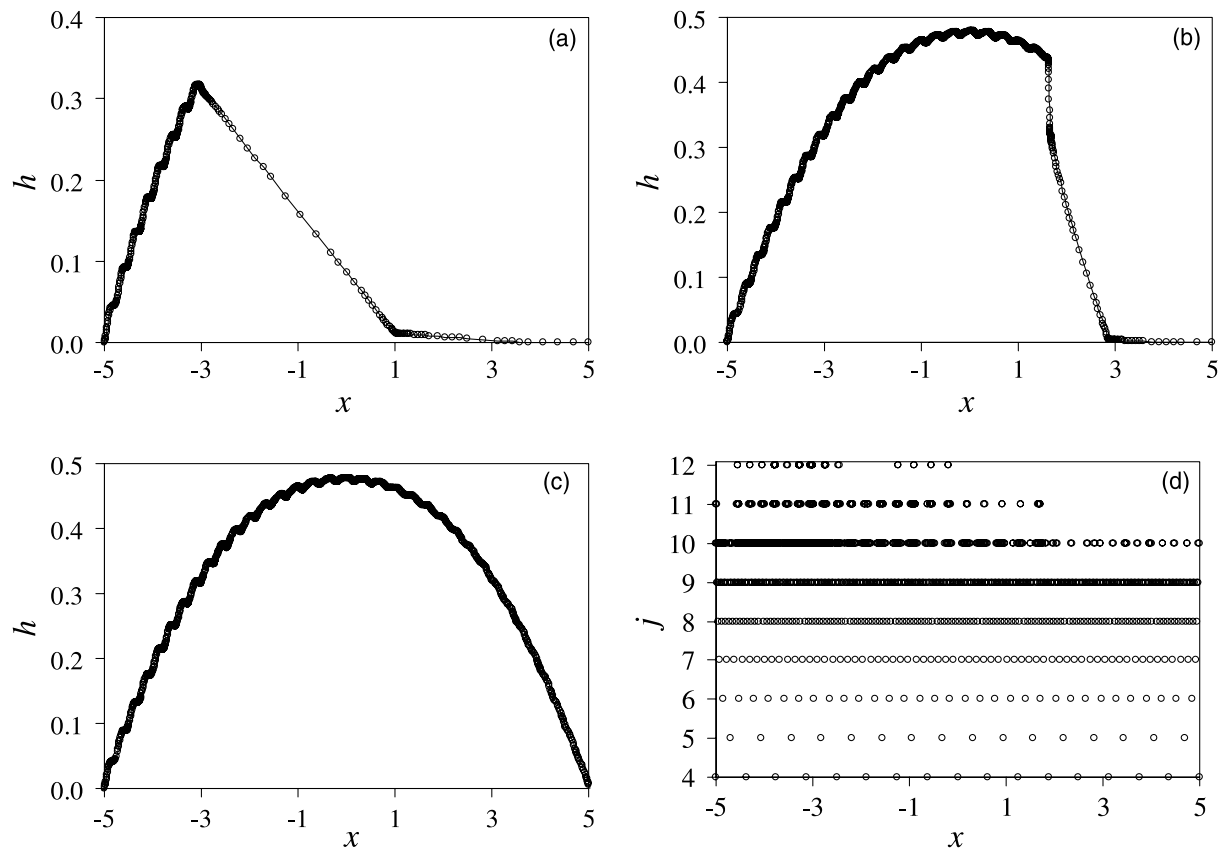

Fig. 14. Solution of the glacier growth with seasonal variations: (a) $t=7.5$, (b) $t=22.5$, and (c) $t=37.5$, (d) distribution of the grid points in terms of spatial location and resolution level at $t=22.5$. The parameters used in the adaptation algorithm are $\varepsilon=10^{-3}$, $N_{\mathrm{R}}=N_{\mathrm{L}}=2, J_{\min }=4$ and $J_{\max }=12$.

served that the oscillations remained with mesh refinement and so they argued that it was a failure of their SD2 scheme.

In Fig. 14(c) the glacier has attained a "steady" profile (except for the small seasonal variations which are always present), as can also be inferred from Fig. 15, showing a small cyclic variation (with a one year period) of the required number of grid points at around 1000.

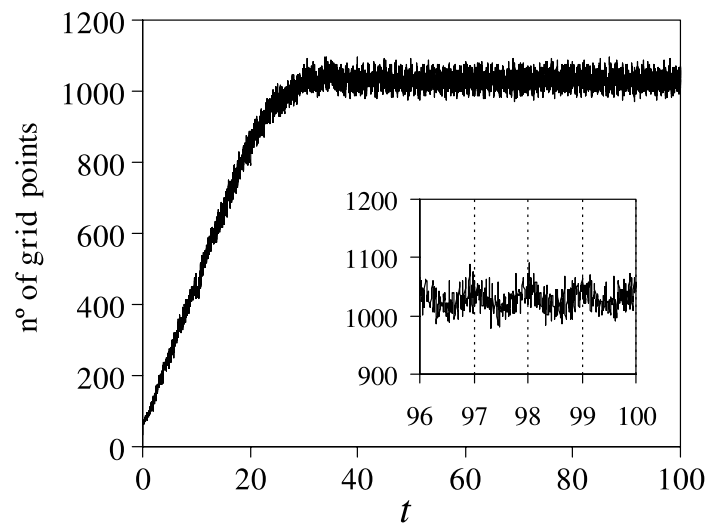

Fig. 15. Number of grid point used by the adaptation algorithm for the solution of the growing glacier with seasonal variations. Adaptation parameters as in Fig. 14. 


\subsection{Double pulse propagation}

Now consider a simple model of double pulse propagation moving in opposite directions, which interact when they cross, according to

$$
\left\{\begin{array}{l}
\frac{\partial u}{\partial t}=-\frac{\partial u}{\partial x}+100 u v \\
\frac{\partial v}{\partial t}=+\frac{\partial v}{\partial x}-100 u v
\end{array}\right.
$$

This simple example illustrates the applicability of the proposed strategy to solve hyperbolic systems of PDEs. The initial and boundary conditions considered were

$$
\begin{aligned}
& u(x, 0)= \begin{cases}1, & 0.1<x<0.2, \\
0, & \text { elsewhere, }\end{cases} \\
& v(x, 0)= \begin{cases}1, & 0.8<x<0.9, \\
0, & \text { elsewhere, }\end{cases} \\
& \left.\frac{\partial u}{\partial x}\right|_{x=1}=\left.\frac{\partial v}{\partial x}\right|_{x=0}=0
\end{aligned}
$$

and the results obtained for $t=0.2,0.325$ and 0.5 are reported in Fig. 16. The corresponding number of grid points used by the adaptation algorithm is plotted in Fig. 17; during the propagation stages, before and after interaction, the number of grid points remains approximately constant (at around 200 and 225), and during the pulse interaction it actually drops. The minimum number of grid points occurs at the moment when the discontinuities of the two pulses coincide (there are only two fronts, instead of four).

\subsection{Euler system of equations}

In this final example the adaptive strategy is tested in the one-dimensional Euler conservation laws for gas dynamics,

$$
\frac{\partial}{\partial t}\left[\begin{array}{l}
\rho \\
\rho u \\
E
\end{array}\right]+\frac{\partial}{\partial x}\left[\begin{array}{c}
\rho u \\
\rho u^{2}+p \\
u(E+p)
\end{array}\right]=0
$$

where $\rho, u$ and $E$ are the gas density, velocity and total energy per unit volume, respectively. The pressure, $p$, is given by

$$
p=(\gamma-1)\left(E-\rho u^{2} / 2\right),
$$

where $\gamma$ is the ratio of specific heats, which takes the usual value of 1.4, a good approximation for air. We solved the benchmark Riemann problem proposed by Sod [25], which consists of the initial data

$$
\left[\begin{array}{c}
\rho \\
\rho u \\
E
\end{array}\right]_{t=0}= \begin{cases}{\left[\begin{array}{lll}
1 & 0 & 2.5
\end{array}\right]^{\mathrm{T}},} & x<0.5 \\
{\left[\begin{array}{lll}
0.125 & 0 & 0.25
\end{array}\right]^{\mathrm{T}},} & x>0.5\end{cases}
$$

with a discontinuity in density and total energy at $x=0.5$.

Several approaches can be found in the literature for the solution of Euler system of equations, either in the context of Riemann solvers [13] or pressure-based algorithms [5].

In this work we selected the semi-discrete central scheme of Kurganov and Tadmor [17], which is an extension to higher-order accuracy of the well-known Lax-Friedrichs scheme [18]. This semi-discrete central 

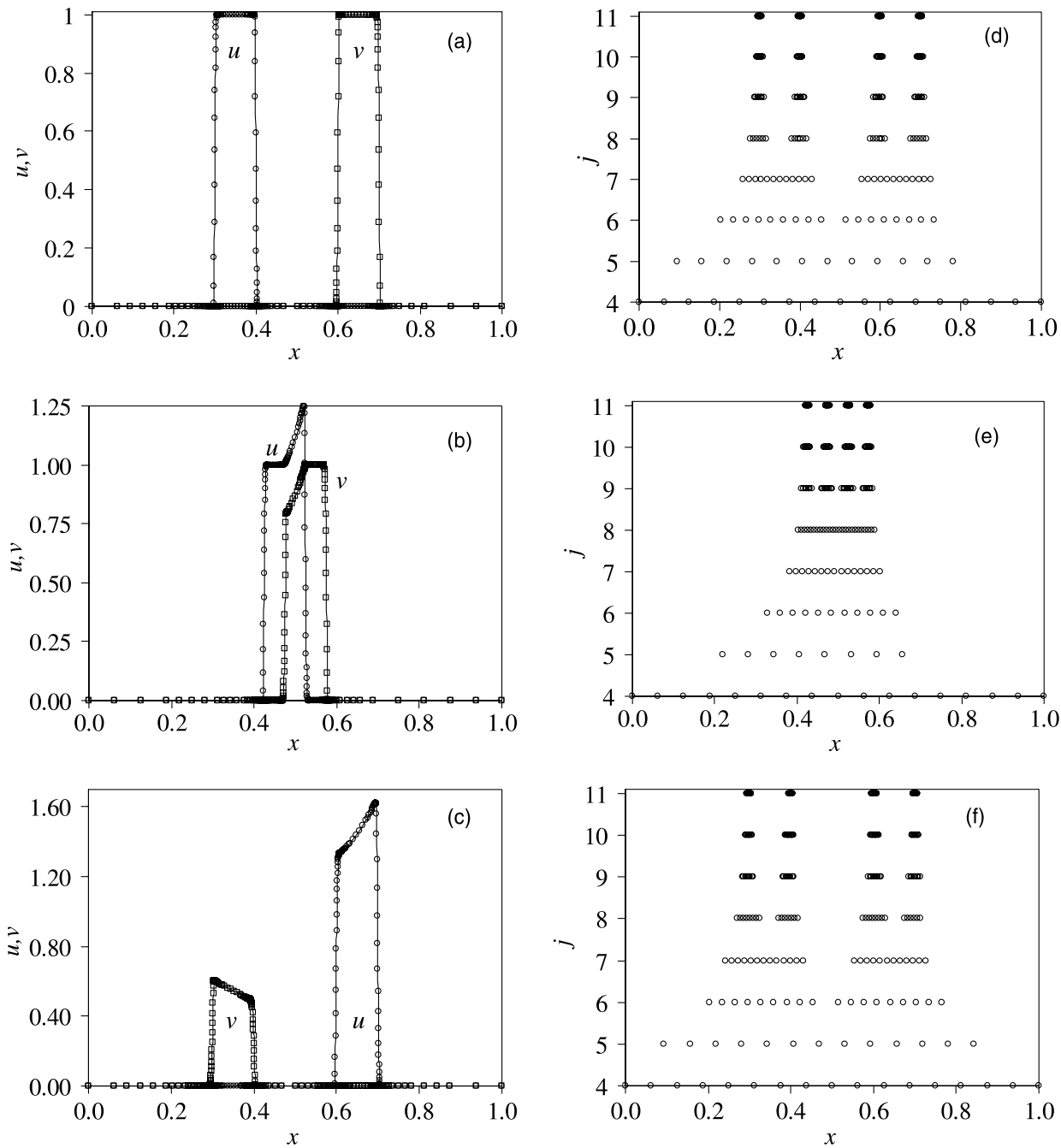

Fig. 16. Solution for the double pulse propagation problem at (a) $t=0.2$; (b) $t=0.325$; and (c) $t=0.5$. Distribution of the grid points in terms of spatial location and resolution level at (d) $t=0.2$; (e) $t=0.325$; and (f) $t=0.5$. The parameters used in the adaptation algorithm are $\varepsilon=10^{-3}, N_{\mathrm{R}}=N_{\mathrm{L}}=2, J_{\min }=4$ and $J_{\max }=11$.

scheme requires the use of a TVD limiter. It is not difficult to modify the SMART scheme so that the resulting piecewise linear formulation in NVSF satisfies TVD constraints, and a recent proposal in that direction was put forth by Song et al. [26], the WACEB high-resolution scheme. In this scheme a normalized face flux is calculated from the expression:

$$
\widehat{F}_{\mathrm{f}}(\mathrm{WACEB})=\max \left[\widehat{F}_{\mathrm{P}}, \min \left(2 \widehat{F}_{\mathrm{P}}, \frac{\widehat{x}_{\mathrm{f}}\left(1-\widehat{x}_{\mathrm{f}}\right)}{\widehat{x}_{\mathrm{P}}\left(1-\widehat{x}_{\mathrm{P}}\right)} \widehat{F}_{\mathrm{P}}+\frac{\widehat{x}_{\mathrm{f}}\left(\widehat{x}_{\mathrm{f}}-\widehat{x}_{\mathrm{P}}\right)}{1-\widehat{x}_{\mathrm{P}}}, 1\right)\right]
$$

which is only different from the SMART formulation (14) for small positive $\widehat{F}_{\mathrm{P}}$ values.

Results for the gas density distribution and the corresponding location of grid points at times $t=0.1$ and 0.2 are presented in Fig. 18. The number of grid points used by the adaptation algorithm is plotted in 


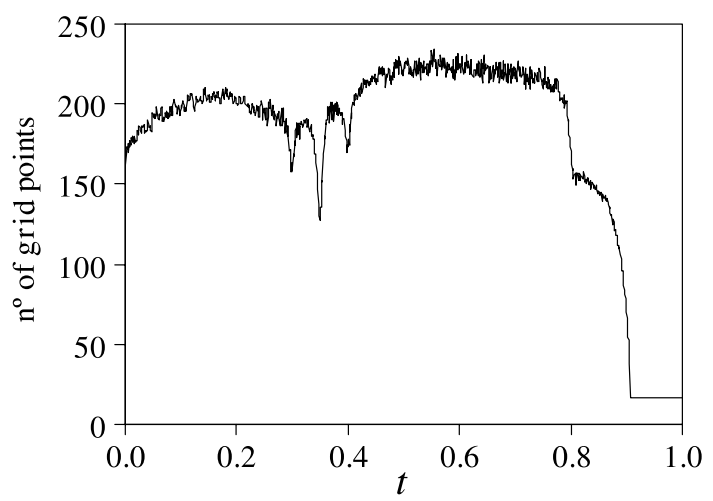

Fig. 17. Time evolution of the number of grid points used by the adaptation algorithm for the solution of the double pulse propagation problem. Adaptation parameters as in Fig. 16.
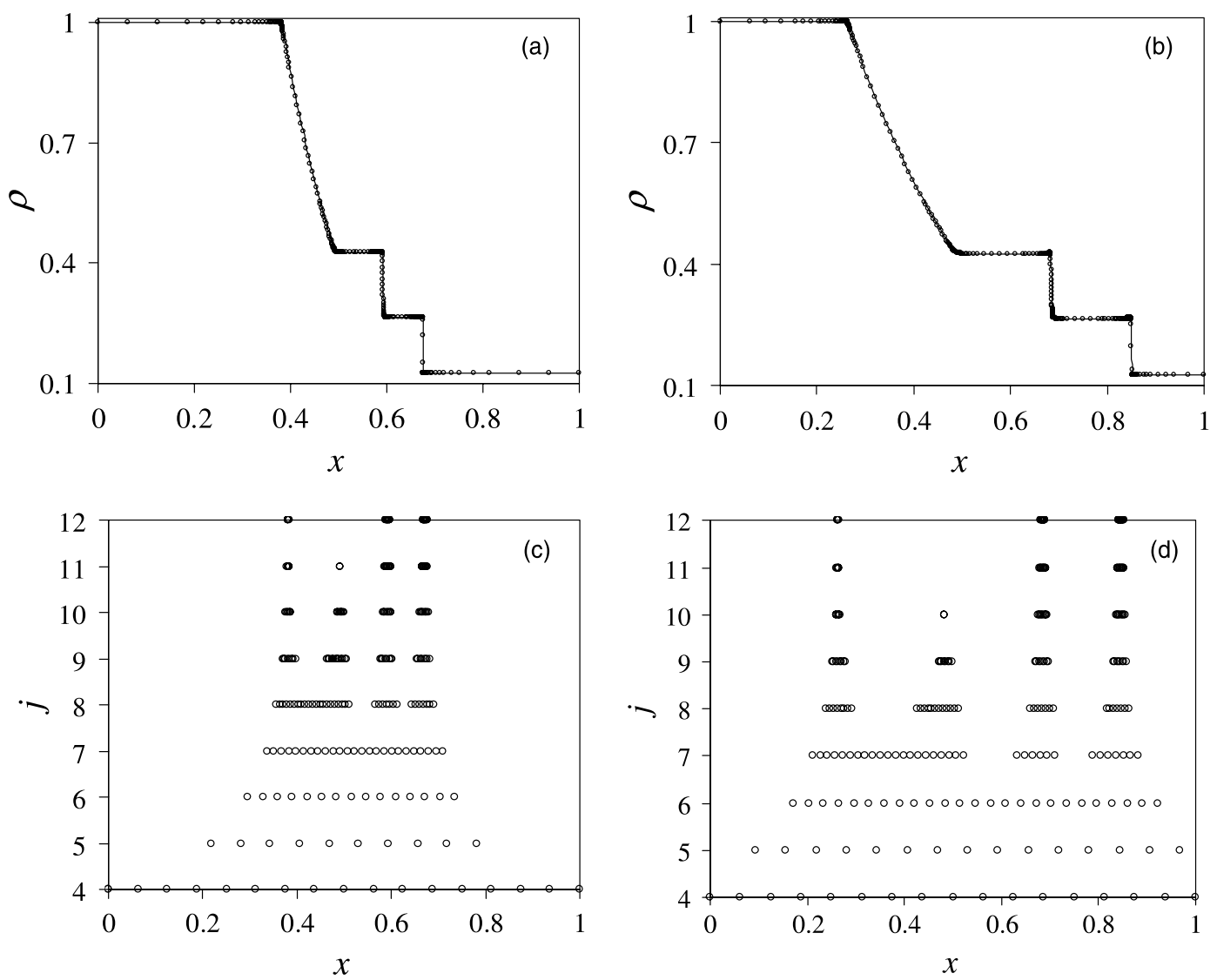

Fig. 18. Computed density profiles from solution of Sod's problem at times (a) $t=0.1$ and (b) $t=0.2$. Distribution of grid points in terms of spatial location and resolution level at (c) $t=0.1$ and (d) $t=0.2$. The parameters used in the adaptation algorithm are $\varepsilon=10^{-4}, N_{\mathrm{R}}=N_{\mathrm{L}}=2, J_{\min }=4$ and $J_{\max }=12$. 


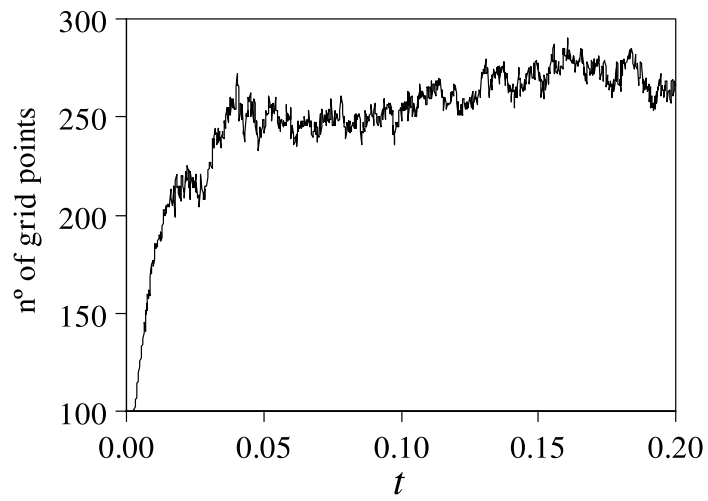

Fig. 19. Time evolution of the number of grid points used by the adaptation algorithm for the solution of Sod's problem with the same parameters as in Fig. 18.

Fig. 19. Once again, the adaptation method proves to be efficient, since a data compression of about $95 \%$ is obtained.

\section{Conclusions}

The novel grid adaptation method presented here, based on the multiresolution approach combined with high-resolution schemes, proved to be very efficient and accurate for the solution of several types of PDEs, ranging from the linear advection equation to the hyperbolic system of Euler equations for gas dynamics. The examples presented demonstrated that a higher grid density is automatically allocated to the regions where the transient solution exhibits sharp gradients, while a coarser grid is used elsewhere, thus making this approach very efficient in terms of memory and CPU time. The adaptive strategy was found to conserve the accuracy of the high-resolution scheme applied to a fixed grid, while reducing the CPU time and memory requirements by more than an order of magnitude, in most cases.

The current simplicity of the method stems from the one-dimensional implementation. The extension to multiple dimensions is possible, and is currently under investigation. Its complexity will increase, but the gains in the performance are expected to be higher than in the one-dimensional case.

\section{Acknowledgements}

M.A. Alves wishes to thank Universidade do Porto and his colleagues at Departamento de Engenharia Química, FEUP, for a temporary leave of absence. The support provided by Fundação Calouste Gulbenkian is also appreciated. The work of Paulo Cruz was funded by FCT (grant BD/21483/99).

\section{References}

[1] S. Bertoluzza, Adaptive wavelet collocation method for the solution of Burgers equation, Transp. Theory Statist. Phys. 25 (1996) $339-352$.

[2] N.N. Carlson, K. Miller, Design and application of a gradient-weighted moving finite element code I: In one dimension, SIAM J. Sci. Comput. 19 (1998) 728-765. 
[3] R. Courant, E. Isaacson, M. Rees, The solution of nonlinear hyperbolic differential equations by finite differences, Commun. Pure Appl. Math. 5 (1952) 243-255.

[4] M.S. Darwish, F. Moukalled, Normalized variable and space formulation methodology for high-resolution schemes, Numer. Heat Transfer Part B 30 (1994) 217-237.

[5] J.H. Ferziger, M. Perić, Computational Methods for Fluid Dynamics, first ed., Springer, Berlin, 1996.

[6] C.A.J. Fletcher, Computational Techniques for Fluid Dynamics, vol. I, second ed., Springer, Berlin, 1991.

[7] C.J. Freitas, Policy statement on the control of numerical accuracy, ASME J. Fluids Engrg. 115 (1993) 339-340.

[8] P.H. Gaskell, A.K.C. Lau, Curvature compensated convective transport: SMART, a new boundedness preserving transport algorithm, Int. J. Numer. Meth. Fluids 8 (1988) 617-641.

[9] S.K. Godunov, A difference scheme for numerical computations of discontinuous solution of hydrodynamic equations, Math. Sbornik 47 (1959) 271-306, in Russian, Translated US Joint Publ. Res. Service, JPRS (1969) 7226.

[10] A. Harten, High resolution schemes for hyperbolic conservation laws, J. Comput. Phys. 49 (1983) 357-393.

[11] A. Harten, B. Engquist, S. Osher, S.R. Chakravarthy, Uniformly high order accurate essentially non-oscillatory schemes, J. Comput. Phys. 71 (1987) 231-303.

[12] A. Harten, Multiresolution representation of data: A general framework, SIAM J. Numer. Anal. 33 (1996) $1205-1256$.

[13] C. Hirsch, Numerical Computation of Internal and External Flows, vol. 2, Wiley, Chichester, 1990.

[14] M. Holmström, Solving hyperbolic PDEs using interpolating wavelets, J. Sci. Comput. 21 (1999) 405-420.

[15] L. Jameson, Wavelet-based grid generation, ICASE Report No. 96-59, NASA Langley Research Center, Hampton, 1996.

[16] K.H. Karlsen, K.-A. Lie, An unconditionally stable splitting scheme for a class of nonlinear parabolic equations, IMA J. Numer. Anal. 19 (1999) 609-635.

[17] A. Kurganov, E. Tadmor, New high-resolution central schemes for nonlinear conservation laws and convection-diffusion equations, J. Comput. Phys. 160 (2000) 241-282.

[18] P.D. Lax, Weak solutions of nonlinear hyperbolic equations and their numerical computation, Commun. Pure Appl. Math. 7 (1954) 159-193.

[19] B.P. Leonard, A stable and accurate convective modeling procedure based on quadratic upstream interpolation, Comput. Meth. Appl. Mech. Engrg. 19 (1979) 59-98.

[20] B.P. Leonard, Locally modified QUICK scheme for highly convective 2-D and 3-D flows, in: C. Taylor, K. Morgan (Eds.), Numerical Methods in Laminar and Turbulent Flow, vol. 5, Pineridge Press, Swansea, UK, 1987, pp. 35-47.

[21] B.P. Leonard, Simple high-accuracy resolution program for convective modelling of discontinuities, Int. J. Numer. Meth. Fluids 8 (1988) 1291-1318.

[22] S. Mallat, Multiresolution approximation and wavelet orthogonal bases of L 2(R), Trans. Am. Math. Soc. 315 (1989) $69-87$.

[23] L.R. Petzold, Automatic selection of methods for solving stiff and nonstiff systems of ordinary differential equations, SIAM J. Sci. Stat. Comput. 4 (1983) 136-148.

[24] W. Shyy, A study of finite difference approximations to steady-state convection dominated flow problems, J. Comput. Phys. 57 (1985) 415-438.

[25] G. Sod, A survey of several finite difference methods for systems of nonlinear hyperbolic conservation laws, J. Comput. Phys. 22 (1978) $1-31$.

[26] B. Song, G.R. Liu, K.Y. Lam, R.S. Amano, On a higher-order bounded discretization scheme, Int. J. Numer. Meth. Fluids 32 (2000) 881-897.

[27] P.K. Sweby, High resolution schemes using flux limiters for hyperbolic conservation laws, SIAM J. Numer. Anal. 21 (1984) $995-$ 1011.

[28] J. Waldén, Filter bank methods for hyperbolic PDEs, J. Numer. Anal. 36 (1999) 1183-1233.

[29] H.-M. Zhou, Wavelet transforms and PDE techniques in image compression, Ph.D. Thesis, Department of Mathematics, UCLA, 2000. 